\title{
Case studies as a research tool
}

In this issue of Quality in Health Care there are two case studies. As these use methodology which may not be familiar to all readers, Louise Fitzgerald, an author of one of these case studies, outlines their structure.

In many fields of knowledge, there is increasing recognition that a range of research methodologies can enrich and extend the available evidence and knowledge base. Understandably, in medical research and in clinical bench sciences, quantitative research methodologies have predominated. In the social sciences, however, qualitative methodologies such as case studies have long been accepted, and indeed developed as useful research tools.

As with any methodology, case studies are best suited to particular forms of enquiry. Under what conditions therefore would one consider using a case study methodology? One appropriate use would be for conducting exploratory research, where the field of enquiry is novel and underresearched. If there is minimal or no foundation of previous research evidence, it is difficult to formulate the critical research questions and useful hypotheses. In these circumstances, case studies can be used to explore and describe the issues within a given context. Appropriate research questions can then be formulated on a foundation of data. For example, there has been limited research on the problems of efficient service delivery in primary care partnerships. We know from some previous research in the private sector that partnerships have unique problems of control and accountability. A case study approach to research, particularly using multiple or comparative case studies would be useful.

Case studies are also useful as research tools in complex and dynamic contexts where it is difficult to isolate variables or where there are multiple, influencing variables. Because many organisational contexts have these characteristics, this is one explanation for the use of case study methods in organisational research. Case studies are a fruitful method to answer the "why" questions in such contexts. For example, in the two cases published in this edition of Quality in Health Care (pages 99 and 108), the authors seek to explore and answer questions about why and under what conditions, clinical professionals decide to adopt an innovation or change their clinical practice. The argument has been posed that if there is strong scientific evidence to support an innovation, then professionals, once they know this evidence, will adopt the innovation. But do we know if this is why clinical professionals change their practice? If it is an influence, is it the only or even the major influence?

Many of the critics of case study methods would simply see a case study as a loosely constructed "story", selected at random (or worse because the researcher is connected to the case in some manner). In many respects this undervalues the approach and certainly underestimates the current stage of development of the case study approach. Yinn, ${ }^{1}$ provides a resource which helps researchers wishing to construct well rounded cases. The selection of the case or cases is a critical stage. Some of the issues of generalisation may be mitigated by a purposeful approach to case selection, using overt selection criteria and comparative cases. If a case or cases is representative in some way, then this must be verifiable. Case studies have several attributes which it may be helpful to emphasise. Firstly, case studies can employ various forms and sources of data. The case can be built up using descriptive statistics and facts drawn from current, secondary sources. These facts can be supplemented with new data produced by interviewing or surveying a range of people who have knowledge to bring to bear on the theme under investigation. Cases may also employ observational data, particularly of interactions such as professional/patients contacts. Secondly, cases can use both historical and real time data. It is frequently important to understand and map the historical development of an issue. This attribute of case studies is vitally important because it allows the researcher to track processes through time. For example, in the case example of glue ear in the article by Dopson et al (page 108), what factors led clinicians to change their already established practice? The processes of discontinuing and changing are likely to be different from the processes of innovation.

The most contentious aspect of case studies as a research tool is probably the analysis phase. Once data have been collected, how can one use them to develop explanatory and descriptive themes which are robust? There are various established methods of data analysis, using interative processes of content analysis to produce analytic themes. Clearly, one of the most significant aspects of analysis is to be able to document the foundational data and exemplifications of these data. More recently, there has been an upsurge in computer software to assist in the scanning and analysis of qualitative data. One of the best known of these-that is, NUDIST software, has been used in the analysis of the data in the Dopson et al case. An equally critical phase after the analysis is the stage of theory building. Reflection on the analytical themes emerging and on other similar and cumulative work enables the researcher to start developing theories and formulating ideas for future research.

LOUISE FITZGERALD

Professor, School of Nursing and Midwifery, City University, London, UK

1 Yinn RK. Case study research: design and methods. London: Sage, 1989. 